\title{
Covariants Vanishing on Totally Decomposable Forms
}

\author{
Emmanuel Briand
}

In honor of Professor Federico Gaeta

\begin{abstract}
We consider the problem of providing systems of equations characterizing the forms with complex coefficients that are totally decomposable, i.e., products of linear forms. Our focus is computational. We present the well-known solution given at the end of the nineteenth century by Brill and Gordan and give a complete proof that their system does vanish only on the decomposable forms. We explore an idea due to Federico Gaeta which leads to an alternative system of equations, vanishing on the totally decomposable forms and on the forms admitting a multiple factor. Last, we give some insight on how to compute efficiently these systems of equations and point out possible further improvements.
\end{abstract}

Mathematics Subject Classification (2000). 15A72, 14Q15.

Keywords. Totally decomposable forms, Brills equations.

\section{Introduction}

Consider the following problem: find "good" systems of algebraic equations that characterize those forms with complex coefficients that are "totally decomposable", that is, products of linear forms. A solution of this problem was provided by Brill and Gordan [9] at the end of the nineteenth century. Their system of equations is obtained from a covariant (afterwards referred to as Brill's covariant) built from simple geometric considerations and classical constructions of the invariant theory of their time. The overall construction is, nevertheless, quite intricate and the systems of equations obtained this way are huge.

I met this problem several years ago when working on my thesis about the diagonal invariants of the symmetric group (a generalization of the symmetric

Emmanuel Briand is supported by the projects MTM2007-64509 (MICINN, Spain) and FQM333 (Junta de Andalucía). 
functions arising naturally in some problems related to systems of polynomial equations). In 1999, Laureano Gonzalez Vega, one my thesis advisors, and MariEmi Alonso introduced me to Professor Gaeta. Besides marvelling me with the elegance of the algebraic geometry à l'italienne, he gave me some interesting suggestions. He designed a particularly simple alternative to Brill's covariant, another covariant that he called the tangential. The tangential of a form $f$ is proportional to $f$ if and only if $f$ is totally decomposable or has a multiple factor. Nevertheless my work took a different direction and I didn't dwell on Gaeta's suggestions.

This paper presents in detail the construction of Gaeta's tangential and provides a formal proof of its fundamental property (Section 3). It also introduces a slight improvement: a new covariant (called Gaeta's covariant), built from the tangential, and which vanishes if and only if $f$ is totally decomposable or has a multiple factor. The construction of Brill's covariant is also recalled, as well as the proof that Brill's covariant vanishes if and only if the form $f$ is totally decomposable (Section 4). Note that Gordan's proof in [9] was not complete since it skipped the case when $f$ had only multiple factors. The proof given here is complete. Last, Section 5 explains how to compute efficiently both Brill's and Gaeta's covariants and suggests some further improvements.

\section{Preliminaries and main results}

Let $\mathbb{K}$ be an algebraically closed field of characteristic zero. Let $n$ and $N$ be positive integers. We consider the forms of degree $n$ with coefficients in $\mathbb{K}$ in the variables $x_{1}, x_{2}, \ldots, x_{N}$ :

$$
f(\mathbf{x})=f\left(x_{1}, \ldots, x_{N}\right)=\sum a_{\omega} \mathbf{x}^{\omega},
$$

where the sum at the right-hand side is carried over all $\omega \in \mathbb{N}^{N}$ whose terms add to $n$, and $\mathbf{x}^{\omega}$ stands for $x_{1}^{\omega_{1}} x_{2}^{\omega_{2}} \cdots x_{N}^{\omega_{N}}$. Among these forms, some decompose totally, i.e., as products of linear forms. The set of the products of linear forms is an irreducible algebraic subvariety that we denote by $\mathcal{D}_{n}\left(\mathbb{K}^{N}\right)$, and call the subvariety of totally decomposable forms.

Problem. Find a system of equations defining set-theoretically $\mathcal{D}_{n}\left(\mathbb{K}^{N}\right)$.

Let us motivate this problem with an application. If one considers a form with rational, or even algebraic, coefficients, and wants to determine whether or not it decomposes totally, the most efficient way to do it is by applying an algorithm of absolute factorization. In this case, there is no hope that a system of equation for $\mathcal{D}_{n}\left(\mathbb{K}^{N}\right)$ would provide a better solution. A different problem is the following: given a family of forms $f_{t_{1}, t_{2}, \ldots}$ depending polynomially on parameters $t_{1}, t_{2}, \ldots$, determine for which values of the parameters the form is totally decomposable. Such a problem appears for instance in [11]. A system of equations defining the subvariety of the totally decomposable forms provides, by specialization, a system of equations in the parameters $t_{1}, t_{2}, \ldots$ whose solutions are the solutions of the original problem. 
Brill [3] and Gordan [9] found a system of equations defining $\mathcal{D}_{n}\left(\mathbb{K}^{N}\right)$ settheoretically. These equations are called Brill's equations in [8], and the name was used afterwards by other authors. Brill's equations are obtained as the coefficients of Brill's covariant, a polynomial function $B: f \mapsto B_{f}$ from the space of forms of degree $n$ over $\mathbb{K}^{N}$ to the space of polynomials in the $3 N$ variables from the following three families:

$$
\mathbf{x}=\left(x_{1}, \ldots, x_{N}\right), \quad \mathbf{y}=\left(y_{1}, \ldots, y_{N}\right), \quad \mathbf{z}=\left(z_{1}, \ldots, z_{N}\right) .
$$

So for each $f$, the polynomial $B_{f}$ writes

$$
B_{f}=\sum b_{\alpha, \beta, \gamma}(f) \mathbf{x}^{\alpha} \mathbf{y}^{\beta} \mathbf{z}^{\gamma},
$$

where the indices $\alpha, \beta, \gamma$ are in $\mathbb{N}^{N}$. Its coefficients $b_{\alpha, \beta, \gamma}(f)$ are polynomial functions of $f$, that is polynomials in the indeterminate coefficients $a_{\omega}$ of $f$ as in (1). Brill's equations are the equations:

$$
b_{\alpha, \beta, \gamma}(f)=0 .
$$

Theorem 1. Let $f$ be a form with coefficients in $\mathbb{K}$. Then $f$ is totally decomposable if and only if $B_{f}$ vanishes identically with respect to $\mathbf{x}, \mathbf{y}$ and $\mathbf{z}$.

Otherwise stated, Brill's equations define $\mathcal{D}_{n}\left(\mathbb{K}^{N}\right)$ set-theoretically.

The name Brill's covariant refers to the following equivariance property of the map $B$. Let $V=\mathbb{K}^{N}$. The target space and source space of $B$ are representations of $S L(V)$ : the source space, the space of forms of degree $n$ on $V$, is the symmetric power $S^{n} V^{*}$. The target space $\mathbb{K}[\mathbf{x}, \mathbf{y}, \mathbf{z}]$ is the symmetric algebra $S^{\bullet}\left(V^{*} \oplus V^{*} \oplus V^{*}\right)$ on three copies of the dual of $V$. This space can be identified with the space of polynomial functions on $V \oplus V \oplus V$. The group $S L(V)$ acts diagonally on this direct sum. This means that for all $M \in S L(V)$ and $\mathbf{u}, \mathbf{v}, \mathbf{w}$ in $V$,

$$
M(\mathbf{u}, \mathbf{v}, \mathbf{w})=(M(\mathbf{u}), M(\mathbf{v}), M(\mathbf{w})) .
$$

With this interpretation, we have for all $f \in S^{n} V^{*}$ and all $M \in S L(V)$ :

$$
B_{f \circ M}=B_{f} \circ M \text {. }
$$

Brill's covariant has another property of invariance, with respect to the group $S L_{2}$, that acts on the first two copies of $V$ in the direct sum $V \oplus V \oplus V$ :

$$
V \oplus V \oplus V \cong(V \oplus V) \oplus V \cong\left(V \otimes \mathbb{K}^{2}\right) \oplus V .
$$

The action of $S L_{2}$ is as follows: for all $\mathbf{u}, \mathbf{v}, \mathbf{w}$ in $V$ and all $\theta=\left[\begin{array}{ll}a & b \\ c & d\end{array}\right] \in S L_{2}$,

$$
\theta(\mathbf{u}, \mathbf{v}, \mathbf{w})=(a \mathbf{u}+b \mathbf{v}, c \mathbf{u}+d \mathbf{v}, \mathbf{w}) .
$$

We have for all $f \in S^{n} V^{*}$ and all $\theta \in S L_{2}$ :

$$
B_{f} \circ \theta=B_{f} .
$$

The invariance property with respect to $S L_{2}$ implies that for all form $f$, the polynomial $B_{f}$ can be written as a polynomial in the variables $\mathbf{z}$ and in the brackets $[i, j]=x_{i} y_{j}-x_{j} y_{i}$ for $1 \leq i<j \leq N$. See Section 5 . 
Let $\mathcal{A}$ be the ring of the $S L(V)$-equivariant polynomial maps from $S^{n} V^{*}$ to $S^{\bullet}(V \oplus V \oplus V)^{*}$ that are invariant under $S L_{2}$ for the above action (2). After what precedes, the map $B$ belongs to $\mathcal{A}$. The $\operatorname{ring} \mathcal{A}$ is endowed with a grading with values in $\mathbb{N}^{3}$ :

$$
\begin{aligned}
\mathcal{A} & \cong\left(S^{\bullet}\left(S^{n} V\right) \otimes S^{\bullet}(V \oplus V \oplus V)^{*}\right)^{S L(V) \times S L_{2}} \\
& \cong \bigoplus_{(d, j, k) \in \mathbb{N}^{3}}\left(S^{d} S^{n} V \otimes\left(S^{j} V^{*} \otimes S^{j} V^{*}\right)^{S L_{2}} \otimes S^{k} V^{*}\right)^{S L(V)} .
\end{aligned}
$$

We will refer to this grading as multidegree. The homogeneous elements of $\mathcal{A}$ of multidegree $(d, j, k)$ are the elements of $\mathcal{A}$ that are homogeneous of degree $d$ in the coefficients of $f$, homogeneous of degree $j$ in the variables $\mathbf{x}$ as well as in the variables $\mathbf{y}$, and homogeneous of degree $k$ in the variables $\mathbf{z}$.

It turns out that Brill's covariant is homogeneous of multidegree $\left(n+1, n, n^{2}-n\right)$.

Gaeta designed another homogeneous element of $\mathcal{A}$, the tangential, $T: f \mapsto$ $T_{f}$ with the following properties.

Theorem 2 (Gaeta). Let $f$ be a form of degree $n$ on $V$. Then:

- For all $\mathbf{u}$ and $\mathbf{v}$ in $V$, the form $T_{f}(\mathbf{u}, \mathbf{v}, \mathbf{z})$ (in the variables $\mathbf{z}$ ) is either 0 or totally decomposable.

- The form $f$ is totally decomposable or admits a multiple factor if and only if $T_{f}$ is a multiple of $f(\mathbf{z})$ in the ring of polynomials in $\mathbf{x}, \mathbf{y}$ and $\mathbf{z}$.

- The form $f$ admits a multiple factor if and only if $T_{f}$ vanishes identically.

That the form $f$ has a multiple factor means that it factorizes into $g h^{2}$ with $g$ and $h$ forms and $h$ non-constant.

Gaeta's tangential is provided by a simple formula: $G_{f}(\mathbf{x}, \mathbf{y}, \mathbf{z})$ is the Sylvester resultant of two binary forms in $(\lambda, \mu)$ : the form $f$ evaluated at $\lambda \mathbf{x}+\mu \mathbf{y}$, and the directional derivative $D_{\mathbf{z}} f$ of $f$ in the direction $\mathbf{z}$, taken at the point $\lambda \mathbf{x}+\mu \mathbf{y}$. The tangential $T$ is a homogeneous element of $\mathcal{A}$ with multidegree $\left(2 n-1, n^{2}-n, n\right)$.

In this paper we improve Gaeta's construction as follows: we build from $T$ a new map $G \in \mathcal{A}$ (homogeneous of the same multidegree as $T$ ) with the property:

Theorem 3. Let $f$ be a form of degree $n$ on $V$. Then $f$ is totally decomposable or admits a multiple factor if and only if $G_{f}$ vanishes identically with respect to $\mathbf{x}, \mathbf{y}$ and $\mathbf{z}$.

We refer to the new polynomial $G_{f}$ as Gaeta's covariant. Its construction, which is simpler than the construction of Brill's covariant, is presented in Section 3. The construction of Brill's covariant is also recalled in Section 4.

\section{Gaeta's tangential and Gaeta's covariant}

In this section we introduce Gaeta's tangential and Gaeta's covariant. A preliminary about classical objects from invariant theory, the polars of a form, is needed. 


\subsection{The polars of a form $f$}

Let $f$ be a form of degree $n$ in $N$ variables. Let $\mathbf{x}=\left(x_{1}, \ldots, x_{N}\right)$ and $\mathbf{y}=$ $\left(y_{1}, \ldots, y_{N}\right)$ be two families of variables. Consider the decomposition:

$$
f(\mathbf{x}+\mathbf{y})=\sum_{i=0}^{n} f^{(i)}(\mathbf{x} ; \mathbf{y})
$$

where $f^{(i)}(x ; y)$ is the component of degree $i$ in the $x$-variables (and thus equivalently: of degree $n-i$ in the $y$-variables $)$. The bihomogeneous forms $f^{(i)}(\mathbf{x} ; \mathbf{y})$ are the polars $^{1}$ of $f$.

Let $\mathbf{u}$ and $\mathbf{v}$ in $V$ with $\mathbf{v}$ non-zero. Then $f^{(1)}(\mathbf{v} ; \mathbf{u})$ is the directional derivative of $f$ at $\mathbf{u}$, in the direction $\mathbf{v}$ :

$$
f^{(1)}(\mathbf{v} ; \mathbf{u})=D_{\mathbf{v}} f(\mathbf{u}) .
$$

In particular, for $\mathbf{u}$ non-zero, the equation $f^{(1)}(\mathbf{z}, \mathbf{u})=0$ is an equation (in $\mathbf{z}$ ) of the tangent space at $[\mathbf{u}]$ for the hypersurface $f=0$ of $\mathbb{P}(V)$.

We conclude with an observation about the polars of a decomposable form. Let $e_{1}, e_{2}, \ldots, e_{n}$ be the elementary symmetric polynomials in $n$ variables.

Lemma 4. Let $f$ be a form of degree $n$. Assume that $f$ is totally decomposable: $f=\ell_{1} \ell_{2} \cdots \ell_{n}$ with $\ell_{1}, \ell_{2}, \ldots, \ell_{n}$ linear.

Then for all $i$ between 0 and $n$, the polar $f^{(i)}(\mathbf{x} ; \mathbf{y})$ is the following homogenized elementary symmetric polynomial:

$$
f^{(i)}(\mathbf{x} ; \mathbf{y})=e_{i}\left(\frac{\ell_{1}(\mathbf{x})}{\ell_{1}(\mathbf{y})}, \frac{\ell_{2}(\mathbf{x})}{\ell_{2}(\mathbf{y})}, \ldots, \frac{\ell_{n}(\mathbf{x})}{\ell_{n}(\mathbf{y})}\right) \cdot f(\mathbf{y})=\sum_{I \subset[n], \# I=i} \prod_{k \in I} \ell_{k}(\mathbf{x}) \prod_{k \notin I} \ell_{k}(\mathbf{y}) .
$$

\subsection{Gaeta's tangential}

Gaeta [7] proposed the following geometric construction to detect totally decomposable forms. Its setting is the projective space $\mathbb{P}(V)$ (recall that $V=\mathbb{K}^{N}$ ). Given a non-zero vector $\mathbf{u} \in V$, the corresponding point in $\mathbb{P}(V)$ will be denoted with $[\mathbf{u}]$.

Let $f$ be a form of degree $n$ on $V$. Assume that $f$ has no multiple factor. A generic line $L$ in $\mathbb{P}(V)$ meets the hypersurface $H_{f}$ of $\mathbb{P}(V)$ defined by $f=0$ at $n$ distinct points, all non-singular on $H_{f}$. Then $f$ is totally decomposable if and only if $H_{f}$ coincides with the union $U(L, f)$ of the tangent hyperplanes of $H_{f}$ at these $n$ intersections.

If $\mathbf{u}$ and $\mathbf{v}$ are non-zero vectors such that $[\mathbf{u}]$ and $[\mathbf{v}] \operatorname{span} L$, then $U(L, f)$ is the zero locus of

$$
\prod_{(\lambda: \mu) \in \mathbb{P}^{1} \mid f(\lambda \mathbf{u}+\mu \mathbf{v})=0} f^{(1)}(\mathbf{z} ; \lambda \mathbf{u}+\mu \mathbf{v}) .
$$

One recognizes in this expression the Sylvester resultant of $f(\lambda \mathbf{u}+\mu \mathbf{v})$ and $f^{(1)}(\mathbf{z} ; \lambda \mathbf{u}+\mu \mathbf{v})$, as binary forms in $(\lambda, \mu)$.

\footnotetext{
${ }^{1}$ Some scalar factor may be needed to fit in with the classical notations.
} 
For any $f \in S^{n} V^{*}$, Gaeta's tangential $T_{f}$ is defined as the following polynomial in $\mathbf{x}, \mathbf{y}$ and $\mathbf{z}$ :

$$
T_{f}=\operatorname{Resultant}_{(\lambda, \mu)}\left(f(\lambda \mathbf{x}+\mu \mathbf{y}), f^{(1)}(\mathbf{z} ; \lambda \mathbf{x}+\mu \mathbf{y})\right) .
$$

Observe that $T$ is a homogeneous element of $\mathcal{A}$ of multidegree $\left(2 n-1, n^{2}-n, n\right)$.

Example 1. Consider the case of quadratic forms. Let $f$ be a quadratic form in $N$ variables. We have:

$$
\begin{aligned}
f(\lambda \mathbf{x}+\mu \mathbf{y}) & =\lambda^{2} f(\mathbf{x})+\lambda \mu f^{(1)}(\mathbf{x} ; \mathbf{y})+\mu^{2} f(\mathbf{y}), \\
f^{(1)}(\mathbf{z} ; \lambda \mathbf{x}+\mu \mathbf{y}) & =\lambda f^{(1)}(\mathbf{x} ; \mathbf{z})+\mu f^{(1)}(\mathbf{y} ; \mathbf{z}) .
\end{aligned}
$$

Therefore,

$$
T_{f}=\left|\begin{array}{ccc}
f(\mathbf{x}) & f^{(1)}(\mathbf{x} ; \mathbf{y}) & f(\mathbf{y}) \\
f^{(1)}(\mathbf{x} ; \mathbf{z}) & f^{(1)}(\mathbf{y} ; \mathbf{z}) & 0 \\
0 & f^{(1)}(\mathbf{x} ; \mathbf{z}) & f^{(1)}(\mathbf{y} ; \mathbf{z})
\end{array}\right|
$$

We now proceed to the proof of Theorem 2 .

Proof of Theorem 2. Let $f$ be a form of degree $n$ on $V$.

That $T_{f}(\mathbf{u}, \mathbf{v}, \mathbf{z})$ is either 0 or totally decomposable for any choice of $\mathbf{u}$ and $\mathbf{v}$ follows from the factorization (3) up to a factor depending only on $\mathbf{u}$ and $\mathbf{v}$.

Consider the assertions:

(i) The form $f$ has a multiple factor.

(ii) The form $T_{f}$ vanishes identically.

(iii) The form $f$ is totally decomposable or admits a multiple factor.

(iv) The form $T_{f}$ is a multiple of $f(\mathbf{z})$.

We should establish on the one hand, the equivalence of (i) and (ii), and, on the other hand, the equivalence of (iii) and (iv).

Let us first establish that (i) and (ii) are equivalent.

Suppose that (i) holds: $f=g h$ with $g$ non-constant and $g$ divides $h$. After the interpretation of the first polar as a directional derivative (see Section 3.1) and Leibnitz' rule for the derivative of a product, we have:

$$
f^{(1)}(\mathbf{z}, \mathbf{x})=g(\mathbf{x}) h^{(1)}(\mathbf{z} ; \mathbf{x})+h(\mathbf{x}) g^{(1)}(\mathbf{z} ; \mathbf{x}) .
$$

In particular $g(\mathbf{x})$ divides also $f^{(1)}(\mathbf{z} ; \mathbf{x})$. Therefore the binary form $g(\lambda \mathbf{x}+\mu \mathbf{y})$ is a non-trivial common factor of $f(\lambda \mathbf{x}+\mu \mathbf{y})$ and $f^{(1)}(\mathbf{z} ; \lambda \mathbf{x}+\mu \mathbf{y})$. We conclude that $T_{f}=0$ after (4), i.e., (ii) holds.

Suppose now that (i) does not hold. Then there exists a line $L$ cutting $H_{f}$ at $n$ distinct points. Let $\mathbf{u}$ and $\mathbf{v}$ be non-zero vectors of $V$ such that $L$ is the line joining $[\mathbf{u}]$ and $[\mathbf{v}]$, and $[\mathbf{u}]$ does not belong to $H_{f}$. Then $T_{f}(\mathbf{u}, \mathbf{v}, \mathbf{u})$ is, up to a non-zero scalar, the discriminant of the binary form $f(\lambda \mathbf{u}+\mu \mathbf{v})$, which has $n$ distinct roots. Therefore this discriminant is non-zero. Since $T_{f}$ does not vanish at $(\mathbf{u}, \mathbf{v}, \mathbf{u})$, it does not vanish identically, i.e., (ii) does not hold.

Let us now establish the equivalence of (iii) and (iv). 
Suppose that (iii) holds. If $f$ admits a multiple factor, then $T_{f}=0$, which is a multiple of $f(\mathbf{z})$. In this case (iv) holds. It remains to show that (iv) also holds when $f$ has no multiple factor and is totally decomposable. This is implied straightforwardly by the following property:

(v) If $\ell$ is a linear factor of $f$, then $\ell(\mathbf{z})$ is also a factor of $T_{f}$.

Let us prove (v). Assume that $f=\ell h$ with $\ell$ linear. After Leibnitz' rule:

$$
f^{(1)}(\mathbf{z} ; \mathbf{x})=\ell(\mathbf{x}) h^{(1)}(\mathbf{z} ; \mathbf{x})+\ell(\mathbf{z}) h(\mathbf{x}) .
$$

Let $\mathbf{w}$ be a vector of $V$ such that $\ell(\mathbf{w})=0$. Then the binary forms $f(\lambda \mathbf{x}+\mu \mathbf{y})$ and $f^{(1)}(\mathbf{w} ; \lambda \mathbf{x}+\mu \mathbf{y})$ have the common factor $\ell(\lambda \mathbf{x}+\mu \mathbf{y})$. It follows that their resultant $T_{f}(\mathbf{x}, \mathbf{y}, \mathbf{w})$ is zero. This shows that the zero locus of $\ell(\mathbf{z})$ is contained in the zero locus of $T_{f}(\mathbf{x}, \mathbf{y}, \mathbf{z})$. Since $\ell(\mathbf{z})$ is irreducible, we conclude that it divides $T_{f}(\mathbf{x}, \mathbf{y}, \mathbf{z})$, as required.

Assume now that (iv) holds: there exists a polynomial $R(\mathbf{x}, \mathbf{y}, \mathbf{z})$ such that $T_{f}(\mathbf{x}, \mathbf{y}, \mathbf{z})=R(\mathbf{x}, \mathbf{y}, \mathbf{z}) \cdot f(\mathbf{z})$. Since $T_{f}$ and $f(\mathbf{z})$ are both homogeneous of degree $n$ in $\mathbf{z}$, the polynomial $R$ has degree 0 in $\mathbf{z}$ and we can write $R=R(\mathbf{x}, \mathbf{y})$. If $R=0$ then $T_{f}=0$. Then $f$ admits a multiple factor. If $R \neq 0$ then there exist vectors $\mathbf{u}$ and $\mathbf{v}$ in $V$ such that $R(\mathbf{u}, \mathbf{v}) \neq 0$. We have:

$$
T_{f}(\mathbf{u}, \mathbf{v}, \mathbf{z})=R(\mathbf{u}, \mathbf{v}) \cdot f(\mathbf{z}) .
$$

Since $T_{f}(\mathbf{u}, \mathbf{v}, \mathbf{z})$ is totally decomposable, so is $f(\mathbf{z})$, i.e., (iii) holds.

The properties of invariance with respect to $S L_{2}$ and covariance with respect to $S L(V)$ of $T_{f}$ follow easily from (4).

That $T_{f}$ is proportional to $f(\mathbf{z})$ could provide a system of equations characterizing the totally decomposable forms (among the forms with no multiple factor) as follows. Decompose:

$$
f(\mathbf{z})=\sum_{\omega} a_{\omega} \mathbf{z}^{\omega}, \quad T_{f}=\sum_{\omega} t_{\omega}(f, \mathbf{x}, \mathbf{y}) \mathbf{z}^{\omega} .
$$

The forms are proportional (as forms in $\mathbf{z}$ ) if and only if all determinants $\left|\begin{array}{ll}a_{\alpha} & a_{\beta} \\ t_{\alpha} & t_{\beta}\end{array}\right|$ vanish identically with respect to $\mathbf{x}$ and $\mathbf{y}$.

We present in what follows how to obtain equations of smaller degree.

\subsection{Gaeta's covariant}

Let us consider the ratio $\delta_{f}=T_{f} / f(\mathbf{z})$. When $f$ is totally decomposable, this ratio $\delta_{f}$ admits a simple expression in term of the linear factors of $f$. We will first derive this expression and next exhibit a polynomial covariant continuation $\Delta$ for $\delta$.

Remember that for any homogeneous binary form $\psi(\lambda, \mu)$ and any family of numbers $a_{1}, b_{1}, a_{2}, b_{2}, \ldots, a_{n}, b_{n}$ :

$$
\operatorname{Resultant}_{(\lambda, \mu)}\left(\prod_{i=1}^{n}\left(a_{i} \lambda+b_{i} \mu\right) ; \psi(\lambda, \mu)\right)=\prod_{i=1}^{n} \psi\left(-b_{i}, a_{i}\right) .
$$


This shows that when $f$ decomposes totally as $\ell_{1} \ell_{2} \cdots \ell_{n}$,

$$
\begin{aligned}
T_{f} & =\prod_{i=1}^{n} f^{(1)}\left(\mathbf{z} ;-\ell_{i}(\mathbf{y}) \mathbf{x}+\ell_{i}(\mathbf{x}) \mathbf{y}\right) \\
& =\prod_{i=1}^{n} \sum_{k_{i}=1}^{n} \ell_{k_{i}}(\mathbf{z}) \prod_{j \neq k_{i}}\left(\ell_{i}(\mathbf{x}) \ell_{j}(\mathbf{y})-\ell_{i}(\mathbf{y}) \ell_{j}(\mathbf{x})\right) \\
& =\prod_{i=1}^{n} \ell_{i}(\mathbf{z}) \prod_{j \neq i}\left(\ell_{i}(\mathbf{x}) \ell_{j}(\mathbf{y})-\ell_{i}(\mathbf{y}) \ell_{j}(\mathbf{x})\right) \\
& =f(\mathbf{z}) \prod_{(i, j) \mid i \neq j}\left|\begin{array}{ll}
\ell_{i}(\mathbf{x}) & \ell_{j}(\mathbf{x}) \\
\ell_{i}(\mathbf{y}) & \ell_{j}(\mathbf{y})
\end{array}\right|
\end{aligned}
$$

Therefore,

$$
\delta_{f}=\prod_{(i, j) \mid i \neq j}\left|\begin{array}{lr}
\ell_{i}(\mathbf{x}) & \ell_{j}(\mathbf{x}) \\
\ell_{i}(\mathbf{y}) & \ell_{j}(\mathbf{y})
\end{array}\right|
$$

We recognize a discriminant. Given a binary form $\phi$ of degree $n$ in the variables $(\lambda, \mu)$ :

$$
\phi(\lambda, \mu)=c_{n} \lambda^{n}+c_{n-1} \lambda^{n-1} \mu+\cdots=\prod_{i=1}^{n}\left(\lambda a_{i}+\mu b_{i}\right),
$$

its discriminant is defined as:

$$
\operatorname{Disc}_{(\lambda, \mu)}(\phi)=\operatorname{Resultant}_{(\lambda, \mu)}\left(\phi ; \frac{\partial \phi}{d \lambda}\right) / c_{n} .
$$

(Note that the sign may differ from other definitions.) It fulfills:

$$
\operatorname{Disc}_{(\lambda, \mu)}(\phi)=\prod_{(i, j) \mid i \neq j}\left|\begin{array}{ll}
a_{i} & a_{j} \\
b_{i} & b_{j}
\end{array}\right| \text {. }
$$

Therefore, for $f$ totally decomposable,

$$
\delta_{f}=\operatorname{Disc}_{(\lambda, \mu)}(f(\lambda \mathbf{x}+\mu \mathbf{y})) .
$$

We now set $\Delta_{f}$ for $\operatorname{Disc}_{(\lambda, \mu)}(f(\lambda \mathbf{x}+\mu \mathbf{y}))$ and we define:

$$
G_{f}=T_{f}-\Delta_{f} \cdot f(\mathbf{z}) .
$$

The polynomial map $\Delta$ is clearly equivariant under $S L(V)$ and invariant under $S L_{2}$. So is $G$, after the above equality (5). Theorem 3 follows from the above computations and Theorem 2.

We conclude this section with a remark: for $\phi(\lambda, \mu)=f(\lambda \mathbf{x}+\mu \mathbf{y})$, we have

$$
\frac{\partial \phi}{d \lambda}=f^{(1)}(\mathbf{x} ; \lambda \mathbf{x}+\mu \mathbf{y}) .
$$

Moreover, the coefficient of $\lambda^{n}$ in $\phi$ is $f(\mathbf{x})$. Therefore

$$
\Delta_{f}=\frac{1}{f(\mathbf{x})} \operatorname{Resultant}_{(\lambda, \mu)}\left(f(\lambda \mathbf{x}+\mu \mathbf{y}) ; f^{(1)}(\mathbf{x} ; \lambda \mathbf{x}+\mu \mathbf{y})\right) .
$$


Comparing with (4) we obtain that:

$$
\Delta_{f}=T_{f}(\mathbf{x}, \mathbf{y}, \mathbf{x}) / f(\mathbf{x}) .
$$

Example 2 (Continuation of Example 1). For $f$ quadratic we have:

$$
T_{f}=\left|\begin{array}{ccc}
f(\mathbf{x}) & f^{(1)}(\mathbf{x} ; \mathbf{y}) & f(\mathbf{y}) \\
f^{(1)}(\mathbf{x} ; \mathbf{z}) & f^{(1)}(\mathbf{y} ; \mathbf{z}) & 0 \\
0 & f^{(1)}(\mathbf{x} ; \mathbf{z}) & f^{(1)}(\mathbf{y} ; \mathbf{z})
\end{array}\right|
$$

The covariant $\Delta_{f}$ is obtained by replacing $\mathbf{z}$ with $\mathbf{x}$ in $T_{f}$, and dividing by $f(\mathbf{x})$. For $f$ quadratic we have $f^{(1)}(\mathbf{x} ; \mathbf{x})=2 f(\mathbf{x})$. We obtain:

$$
\Delta_{f}=4 f(\mathbf{x}) f(\mathbf{y})-\left(f^{(1)}(\mathbf{x} ; \mathbf{y})\right)^{2} .
$$

Expanding $T_{f}-\Delta_{f} \cdot f(\mathbf{z})$ we get:

$$
\begin{array}{r}
G_{f}=f(\mathbf{x})\left(f^{(1)}(\mathbf{y} ; \mathbf{z})\right)^{2}+f(\mathbf{y})\left(f^{(1)}(\mathbf{x} ; \mathbf{z})\right)^{2}-f^{(1)}(\mathbf{x} ; \mathbf{y}) f^{(1)}(\mathbf{x} ; \mathbf{z}) f^{(1)}(\mathbf{y} ; \mathbf{z}) \\
-4 f(\mathbf{x}) f(\mathbf{y}) f(\mathbf{z})+f(\mathbf{z})\left(f^{(1)}(\mathbf{x} ; \mathbf{y})\right)^{2} .
\end{array}
$$

This can be presented as follows:

$$
G_{f}=-4\left|\begin{array}{ccc}
f(\mathbf{x}) & f^{(1)}(\mathbf{x} ; \mathbf{y}) / 2 & f^{(1)}(\mathbf{x} ; \mathbf{z}) / 2 \\
f^{(1)}(\mathbf{x} ; \mathbf{y}) / 2 & f(\mathbf{y}) & f^{(1)}(\mathbf{y} ; \mathbf{z}) / 2 \\
f^{(1)}(\mathbf{x} ; \mathbf{z}) / 2 & f^{(1)}(\mathbf{y} ; \mathbf{z}) / 2 & f(\mathbf{z})
\end{array}\right|
$$

\section{Brill's covariant}

In this section we report on Gordan's geometric presentation [9] of Brill's covariant. Such a presentation is already provided by the monograph [8], and of course by Gordan's original text [9], but in neither case the proof of Theorem 1 is complete.

As a preliminary, a classical construction in invariant theory, needed in the sequel, is presented.

\subsection{The apolar covariant}

Consider a pair of binary forms of degree $n$ :

$$
\phi(\lambda, \mu)=\sum \phi_{i, j} \lambda^{i} \mu^{j}, \quad \psi(\lambda, \mu)=\sum \psi_{i, j} \lambda^{i} \mu^{j} .
$$

Their apolar form is the scalar:

$$
\begin{aligned}
\operatorname{Apo}_{(\lambda, \mu)}(\phi, \psi) & =\phi\left(-\frac{\partial}{d \mu}, \frac{\partial}{d \lambda}\right) \psi(\lambda, \mu), \\
& =\sum_{i+j=n}(-1)^{i} i ! j ! \phi_{i, j} \psi_{j, i} .
\end{aligned}
$$

One says that $\phi$ and $\psi$ are apolar to each other if their apolar form is zero. The apolar form is a combinant. This means that it is an invariant under the two actions of $S L_{2}$ : 
- Under change of variables: for all $\theta \in S L_{2}$ and all binary forms $\phi$ and $\psi$ of degree $n$,

$$
\operatorname{Apo}_{(\lambda, \mu)}(\phi \circ \theta, \psi \circ \theta)=\operatorname{Apo}_{(\lambda, \mu)}(\phi, \psi) .
$$

- Under linear combinations of the two quadratic forms: for all $\theta=\left(\begin{array}{ll}a & b \\ c & d\end{array}\right) \in S L_{2}$ and all binary forms $\phi$ and $\psi$ of degree $n$,

$$
\operatorname{Apo}_{(\lambda, \mu)}(a \phi+b \psi, c \phi+d \psi)=\operatorname{Apo}_{(\lambda, \mu)}(\phi, \psi) .
$$

The apolar form was very well known at the end of the nineteenth century. The following properties were familiar at the time.

Lemma 5. Let $\phi$ be a binary form of degree $n$.

- Let $\ell_{1} \ell_{2} \cdots \ell_{n}$ be a factorization of $\phi$ as a product of linear forms. Then all the linear combinations of $\ell_{1}^{n}, \ell_{2}^{n}, \ldots, \ell_{n}^{n}$ are apolar to $\phi$.

- Let $\ell$ be a linear form. Assume that $\ell^{n}$ is apolar to $\phi$. Then $\ell$ divides $\phi$.

Clebsch's transfer principle is a classical method to produce from each invariant $I_{(\lambda, \mu)}$ of pairs of binary forms in $(\lambda, \mu)$ of degree $n$ a covariant $C_{I, \mathbf{x}}$ of pairs of forms in $\mathbf{x}=\left(x_{1}, x_{2}, \ldots, x_{N}\right)$ of degree $n$ :

$$
C_{I, \mathbf{x}}(f, g)(\mathbf{x}, \mathbf{y})=I_{(\lambda, \mu)}(f(\lambda \mathbf{x}+\mu \mathbf{y}), g(\lambda \mathbf{x}+\mu \mathbf{y})) .
$$

If $I_{(\lambda, \mu)}$ is a combinant then $C_{I, \mathbf{x}}$ is not only invariant under $S L_{N}$ acting by changes of variables, but also under $S L_{2}$ acting by linear combination of the families of variables $\mathbf{x}$ and $\mathbf{y}$. That is, $C_{I, \mathbf{x}}$ belongs to the algebra $\mathcal{A}$ introduced in Section 2 .

Applying Clebsch's transfer principle to the apolar form produces the apolar covariant of pairs $(f, g)$ of $N$-ary forms of degree $n$ :

$$
C_{\text {Apo }, \mathbf{x}}(f, g)(\mathbf{x}, \mathbf{y})=\sum_{k=0}^{n}(-1)^{k} k !(n-k) ! f^{(k)}(\mathbf{x} ; \mathbf{y}) g^{(n-k)}(\mathbf{x} ; \mathbf{y}) .
$$

As in the case of binary forms, we will say that the $N$-ary forms $f$ and $g$ are apolar when their apolar covariant vanishes.

In the proof of Proposition 2.10 of [8], the apolar covariant is interpreted in the language of modern representation theory. It corresponds, up to a scalar factor, to the projection from $S^{n}\left(V^{*}\right) \otimes S^{n}\left(V^{*}\right)$ to the Weyl module $S^{(n, n)}\left(V^{*}\right)$ in the decomposition

$$
S^{n}\left(V^{*}\right) \otimes S^{n}\left(V^{*}\right)=\bigoplus_{k=0}^{n} S^{(n+k, n-k)}\left(V^{*}\right) .
$$

and is called there Young's vertical multiplication.

Suppose that $f$ and $g$ are forms of degree $n$ on $V=\mathbb{K}^{N}$ and $\mathbf{u}$ and $\mathbf{v}$ are non-proportional vectors in $V$. Then $f$ and $g$ restrict to binary forms on the plane spanned by $\mathbf{u}$ and $\mathbf{v}$. That $C_{\mathrm{Apo}, \mathbf{x}}(f, g)(\mathbf{u}, \mathbf{v})$ vanishes means that these restrictions are apolar. The following properties of the apolar covariant are straightforwardly deduced from this remark and the corresponding properties of the apolar form (Lemma 5). 
Lemma 6. Let $f$ be a form of degree $n$ on $V$.

- Assume that $f$ is totally decomposable, factorizing in a product of linear forms as $f=\ell_{1} \ell_{2} \cdots \ell_{n}$. Then, for all linear combinations $g$ of $\ell_{1}^{n}, \ell_{2}^{n}, \ldots, \ell_{n}^{n}$, the apolar covariant $C_{\mathrm{Apo}, \mathbf{x}}(f, g)$ vanished identically.

- Let $\ell$ be a linear form on $V$. If $C_{\mathrm{Apo}, \mathbf{x}}\left(f, \ell^{n}\right)$ vanishes identically then $\ell d i$ vides $f$.

\subsection{Gordan's presentation}

Let $f$ be a totally decomposable form of degree $n$ that decomposes as a product of linear forms as $f=\ell_{1} \ell_{2} \cdots \ell_{n}$. The polars of $f$, after suitable normalization, will be interpreted as the elementary symmetric polynomials in scalar multiples of $\ell_{1}(\mathbf{x}), \ell_{2}(\mathbf{x}), \ldots, \ell_{n}(\mathbf{x})$. We will be able to compute the corresponding $n$th symmetric power sum, which, after Lemma 6 , will be apolar to $f$. The vanishing of the apolar covariant of $f$ and the $n$th symmetric power sum will therefore be a necessary condition for total decomposability. This condition will be shown to be also sufficient.

After Lemma 4, for all $i$ between 0 and $n$ we have:

$$
f^{(i)}(\mathbf{x} ; \mathbf{z})=f(\mathbf{z}) \cdot e_{i}\left(\frac{\ell_{1}(\mathbf{x})}{\ell_{1}(\mathbf{z})} ; \cdots ; \frac{\ell_{n}(\mathbf{x})}{\ell_{n}(\mathbf{z})}\right) .
$$

Let us clear all denominators by multiplying with $f(\mathbf{z})^{i-1}$ :

$$
f(\mathbf{z})^{i-1} f^{(i)}(\mathbf{x} ; \mathbf{z})=e_{i}\left(\frac{f(\mathbf{z})}{\ell_{1}(\mathbf{z})} \ell_{1}(\mathbf{x}) ; \cdots ; \frac{f(\mathbf{z})}{\ell_{n}(\mathbf{z})} \ell_{n}(\mathbf{x})\right) .
$$

The polynomials $f(\mathbf{z})^{i-1} f^{(i)}(\mathbf{x} ; \mathbf{z})$ are therefore the elementary symmetric polynomials in the roots of

$$
\sum_{i=0}^{n} f^{(i)}(\mathbf{x} ; \mathbf{z}) f(\mathbf{z})^{i-1}(-1)^{i} t^{n-i}=f(t \mathbf{z}-f(\mathbf{z}) \mathbf{x}) / f(\mathbf{z}) .
$$

The $n$th symmetric power sum $p_{n}$ in $n$ variables has a unique representation as a polynomial in the elementary symmetric polynomials. Let $P_{n}$ be the polynomial such that

$$
p_{n}=P_{n}\left(e_{1}, e_{2}, \ldots, e_{n}\right) .
$$

For any form $f$ of degree $d \leq n$ define:

$$
Q_{n}(f)=P_{n}\left(f^{(1)}(\mathbf{x} ; \mathbf{z}), f(\mathbf{z}) f^{(2)}(\mathbf{x} ; \mathbf{z}), \ldots, f(\mathbf{z})^{d-1} f^{(d)}(\mathbf{x} ; \mathbf{z}), 0, \ldots, 0\right) .
$$

Then $Q_{n}(f)$ is a covariant of the forms $f$. As a consequence, so is the apolar covariant of $f$ and $Q_{n}(f)$. Brill's covariant is this polynomial:

$$
B_{f}=C_{\mathrm{Apo}, \mathbf{x}}\left(f, Q_{n}(f)\right) .
$$

Example 3. For $f$ quadratic we have:

$$
p_{2}=e_{1}^{2}-2 e_{2}
$$


Therefore,

$$
Q_{2}(f)=\left(f^{(1)}(\mathbf{x} ; \mathbf{z})\right)^{2}-2 f(\mathbf{x}) f(\mathbf{z})
$$

Setting $g$ for $Q_{2}(f)$ we have $g^{(0)}(\mathbf{x} ; \mathbf{y})=g(\mathbf{y}), g^{(2)}(\mathbf{x} ; \mathbf{y})=g(\mathbf{x})$ and:

$$
g^{(1)}(\mathbf{x} ; \mathbf{y})=2\left(f^{(1)}(\mathbf{x} ; \mathbf{z}) f^{(1)}(\mathbf{y} ; \mathbf{z})-f^{(1)}(\mathbf{x} ; \mathbf{y}) f(\mathbf{z})\right) .
$$

Therefore,

$$
\begin{aligned}
B_{f}= & 2 f(\mathbf{x})\left(\left(f^{(1)}(\mathbf{y} ; \mathbf{z})\right)^{2}-2 f(\mathbf{y}) f(\mathbf{z})\right) \\
& -2 f^{(1)}(\mathbf{x} ; \mathbf{y})\left(f^{(1)}(\mathbf{x} ; \mathbf{z}) f^{(1)}(\mathbf{y} ; \mathbf{z})-f^{(1)}(\mathbf{x} ; \mathbf{y}) f(\mathbf{z})\right) \\
& +2 f(\mathbf{y})\left(\left(f^{(1)}(\mathbf{x} ; \mathbf{z})\right)^{2}-2 f(\mathbf{x}) f(\mathbf{z})\right)
\end{aligned}
$$

This can be presented as:

$$
B_{f}=-\left|\begin{array}{ccc}
2 f(\mathbf{x}) & f^{(1)}(\mathbf{x} ; \mathbf{y}) & f^{(1)}(\mathbf{x} ; \mathbf{z}) \\
f^{(1)}(\mathbf{x} ; \mathbf{y}) & 2 f(\mathbf{y}) & f^{(1)}(\mathbf{y} ; \mathbf{z}) \\
f^{(1)}(\mathbf{x} ; \mathbf{z}) & f^{(1)}(\mathbf{y} ; \mathbf{z}) & 2 f(\mathbf{z})
\end{array}\right|
$$

The covariant $Q_{n}$ is a homogeneous element of the $\operatorname{ring} \mathcal{A}$ (see Section 2) with multidegree $\left(n, n, n^{2}-n\right)$. As a consequence, $B$ is homogeneous of multidegree $\left(n+1, n, n^{2}-n\right)$.

By construction, the identical vanishing of $B_{f}$ is a necessary condition for total decomposability of $f$. Let us show now that the identical vanishing of $B_{f}$ is also a sufficient condition for total decomposability of $f$. The proof rests on the following lemma.

Lemma 7. Let $f$ be a form of degree $n$ on $V$ whose Brill covariant vanishes identically. Let $[\mathbf{w}]$ be a smooth point on the reduced hypersurface $H_{f}$ of $\mathbb{P}(V)$ defined by $f=0$. Then the irreducible component of $H_{f}$ containing $[\mathbf{w}]$ is a hyperplane.

Observe that, under the hypotheses of the Lemma, there is a unique irreducible factor $g$ of $f$ such that $g(\mathbf{w})=0$, and the linear form $g(\mathbf{x} ; \mathbf{w})$ is non-zero.

Admit the lemma for now. Suppose that $B_{f}$ vanishes identically. Any irreducible component $\Gamma$ of $H_{f}$ has a smooth point [w] not contained in any other irreducible component. After the lemma, $\Gamma$ is necessarily an hyperplane. Therefore $f$ is totally decomposable.

Theorem 1 will therefore be proved, as soon as we have proved Lemma 7 .

Proof of Lemma 7. Let $g=0$ be an equation for the irreducible component of $H_{f}$ containing $[\mathbf{w}]$.

Consider first the simplest case: the factor $g$ of $f$ is not multiple. Since $B_{f}$ vanishes identically, in particular $B_{f}(\mathbf{x}, \mathbf{y}, \mathbf{w})$ vanishes identically with respect to $\mathbf{x}$ and $\mathbf{y}$. The evaluation at $\mathbf{z}=\mathbf{w}$ of the polynomial defined in (6) is:

$$
t^{n}-t^{n-1} f^{(1)}(\mathbf{x}, \mathbf{w}) \text {. }
$$


Remember that $f^{(1)}(\mathbf{x}, \mathbf{w})=0$ is an equation (in $\mathbf{x}$ ) for the tangent space of $f$ at $[\mathbf{w}]$. In particular, since $[\mathbf{w}]$ is non-singular on $H_{f}$ and cancels no multiple factor of $f$, the linear form $f^{(1)}(\mathbf{x}, \mathbf{w})$ is non-zero. Therefore, the roots of $f(t \mathbf{w}-f(\mathbf{w}) \mathbf{x})$ are 0 (with multiplicity $n-1$ ) and $f^{(1)}(\mathbf{x}, \mathbf{w})$ (with multiplicity 1 ). Thus the corresponding $n$th symmetric power sum is $Q_{n}(f)(\mathbf{x}, \mathbf{w})=\left(f^{(1)}(\mathbf{x}, \mathbf{w})\right)^{n}$. It is apolar to $f$ since $B_{f}(\mathbf{x}, \mathbf{y}, \mathbf{w})$ is zero. This implies by Lemma 6 that the linear form $f^{(1)}(\mathbf{x}, \mathbf{w})$ divides $f$. Therefore the hyperplane $H$ with equation $f^{(1)}(\mathbf{x}, \mathbf{w})=0$ is an irreducible component of $H_{f}$ containing $[\mathbf{w}]$. Note that since $[\mathbf{w}]$ is smooth on $H_{f}$ it cannot belong to any other component.

The case when the irreducible factor $g$ of $f$ is multiple is more complicated ${ }^{2}$. First one establishes the identity:

$$
Q_{n}(f)=f_{1}(z)^{n} Q_{n}\left(f_{2}\right)+f_{2}(z)^{n} Q_{n}\left(f_{1}\right)
$$

for $f=f_{1} f_{2}$. This follows from:

$$
\begin{aligned}
& f(t \mathbf{z}-f(\mathbf{z}) \mathbf{x}) / f(\mathbf{z}) \\
& =f_{1}\left(t \mathbf{z}-f_{1}(\mathbf{z})\left(f_{2}(\mathbf{z}) \mathbf{x}\right)\right) / f_{1}(\mathbf{z}) \cdot f_{2}\left(t \mathbf{z}-f_{2}(\mathbf{z})\left(f_{1}(\mathbf{z}) \mathbf{x}\right)\right) / f_{2}(\mathbf{z}) .
\end{aligned}
$$

From (7) one deduces that for any two forms $f_{1}$ and $f_{2}$ :

$$
Q_{n}\left(f_{1}^{k} f_{2}\right)=f_{1}(\mathbf{z})^{n(k-1)}\left(k f_{2}(\mathbf{z})^{n} Q_{n}\left(f_{1}\right)+f_{1}(\mathbf{z})^{n} Q_{n}\left(f_{2}\right)\right) .
$$

Assume that $f=g^{k} h$, where $g$ does not divide $h$, and, as before, $g$ is the unique irreducible factor of $f$ vanishing at $\mathbf{w}$. Then:

$$
\begin{aligned}
B_{f} & =C_{\mathrm{Apo}, \mathbf{x}}\left(f, Q_{n}(f)\right) \\
& =g(\mathbf{z})^{n(k-1)}\left(k h(\mathbf{z})^{n} C_{\mathrm{Apo}, \mathbf{x}}\left(f, Q_{n}(g)\right)+g(\mathbf{z})^{n} C_{\mathrm{Apo}, \mathbf{x}}\left(f, Q_{n}(h)\right)\right) .
\end{aligned}
$$

Since $B_{f}$ vanishes identically, so does

$$
k h(\mathbf{z})^{n} C_{\mathrm{Apo}, \mathbf{x}}\left(f, Q_{n}(g)\right)+g(\mathbf{z})^{n} C_{\mathrm{Apo}, \mathbf{x}}\left(f, Q_{n}(h)\right) .
$$

Evaluating at $\mathbf{z}=\mathbf{w}$ we get:

$$
0=k h(\mathbf{w})^{n} C_{\mathrm{Apo}, \mathbf{x}}\left(f(\mathbf{x}), Q_{n}(g)(\mathbf{x}, \mathbf{w})\right) .
$$

We have $h(\mathbf{w}) \neq 0$. Therefore

$$
0=C_{\mathrm{Apo}, \mathbf{x}}\left(f(\mathbf{x}), Q_{n}(g)(\mathbf{x}, \mathbf{w})\right) .
$$

We can conclude as in the first case that $g^{(1)}(\mathbf{x} ; \mathbf{w})$ divides $f$.

\footnotetext{
${ }^{2}$ This difficulty has been ignored by Gordan [9] as well as in the account of the construction of Brill's covariant given in [8]. The proof given in these texts for Theorem 1 is incomplete, since it does not rule out the possibility that Brill's covariant vanish for non-totally decomposable forms whose factors are all multiples.
} 


\section{Computations in the ring $\mathcal{A}$}

Let $C$ be either Gaeta's covariant $G$ or Brill's covariant $B$ for forms of degree $n$ in $N$ variables. We have an expansion:

$$
C=\sum_{\alpha, \beta, \gamma} c_{\alpha, \beta, \gamma} \mathbf{x}^{\alpha} \mathbf{y}^{\beta} \mathbf{z}^{\gamma} .
$$

The functions $c_{\alpha, \beta, \gamma}$ are homogeneous polynomials of the same degree in the coefficients $a_{\omega}$ of $f$. The system of all equations $c_{\alpha, \beta, \gamma}(f)=0$ defines set-theoretically:

- $\mathcal{D}_{n}\left(\mathbb{K}^{N}\right)$ for $C=B$

- the union of $\mathcal{D}_{n}\left(\mathbb{K}^{N}\right)$ with the set of forms admitting a multiple factor for $C=G$.

Consider the following problem:

Problem. Compute a linear basis for the linear span $L(C, n, N)$ of the polynomials $c_{\alpha, \beta, \gamma}$.

Computing directly the covariant $C$ and next extracting the coefficients, one will meet two difficulties:

- size: we are computing the huge object $C$, to extract from it smaller objects (its coefficients). We will recall in 5.1 how to compute sequentially the coefficients without computing the whole covariant $C$.

- redundancy: many coefficients $c_{\alpha, \beta, \gamma}$ are linear combinations of the others. This is partly explained by the invariance of $C$ under $S L_{2}$ combining the variables $\mathbf{x}$ and $\mathbf{y}$. See 5.2 and 5.5 .

\subsection{Computing sequentially the coefficients from the source}

Write for each exponent $\gamma$ of the variables $\mathbf{z}$ in $C$ :

$$
C_{\gamma}=\sum_{\alpha, \beta} c_{\alpha, \beta, \gamma} \mathbf{x}^{\alpha} \mathbf{y}^{\beta}
$$

We have $C=\sum_{\gamma} C_{\gamma} \mathbf{z}^{\gamma}$. As explained in [2], the coefficients $C_{\gamma}$ can be computed sequentially from only one of them (the source of $C$ ). This is the consequence of the $S L_{N}$-equivariance property of $C$. We recall the formulas presented in [2].

For each $i$ between 1 and $N$, let $\xi_{i} \in \mathbb{N}^{N}$ be the vector whose all coordinates are zero, except the one in position $i$, being equal to 1 :

$$
\xi_{1}=(1,0,0, \ldots, 0), \quad \xi_{2}=(0,1,0, \ldots, 0), \ldots
$$

For $j$ between 2 and $N$ let $\Delta_{j}$ be the following operator, acting on the polynomials in the coefficients $a_{\omega}$ of $f$ as defined in (1):

$$
\Delta_{j}=\sum_{\omega \text { s.t. } \omega_{1}>0}\left(1+\omega_{j}\right) a_{\omega+\xi_{j}-\xi_{1}} \frac{\partial}{d a_{\omega}} .
$$

Then the following relation holds for the covariant $C$ (see [2]):

$$
\Delta_{j} C=x_{1} \frac{\partial C}{d x_{j}}+y_{1} \frac{\partial C}{d y_{j}}+z_{1} \frac{\partial C}{d z_{j}} .
$$


Extracting the coefficient of $\mathbf{z}^{\gamma+\xi_{j}-\xi_{1}}$ we get:

$$
\Delta_{j} C_{\gamma-\xi_{j}+\xi_{1}}=x_{1} \frac{\partial C_{\gamma-\xi_{j}+\xi_{1}}}{d x_{j}}+y_{1} \frac{\partial C_{\gamma-\xi_{j}+\xi_{1}}}{d y_{j}}+\gamma_{j} C_{\alpha, \beta, \gamma}
$$

Isolate $C_{\gamma}$ :

$$
\gamma_{j} C_{\gamma}=\Delta_{j} C_{\gamma-\xi_{j}+\xi_{1}}-x_{1} \frac{\partial C_{\gamma-\xi_{j}+\xi_{1}}}{d x_{j}}-y_{1} \frac{\partial C_{\gamma-\xi_{j}+\xi_{1}}}{d y_{j}} .
$$

One uses this relation to compute the coefficients $C_{\gamma}$ according to decreasing values of $\gamma$ with respect to lexicographical ordering on $\mathbb{N}^{N}$. Let $k$ be the degree of $C$ in the variables $\mathbf{z}$. As initial values only the coefficient $C_{(k, 0,0, \ldots, 0)}$ is needed. We call this coefficient the source ${ }^{3}$ of the covariant and denote it with Source $(C)$. Note that Source $(C)$ is the evaluation of $C$ at $\mathbf{z}=(1,0,0, \ldots, 0)$. For $C$ equal to $B, T$ or $G$, it is a much smaller object than the whole covariant.

Since we are not interested in the precise value of the covariant $C$, but only in the space $L(C, n, N)$, we do not need to take into account the term $-x_{1} \frac{\partial C_{\gamma}}{d x_{j}}-y_{1} \frac{\partial C_{\gamma}}{d y_{j}}$ in (8). Indeed,

Proposition 8. Let $C$ be an element of $\mathcal{A}$, homogeneous of degree $d$ in the variables $\mathbf{z}$. Then $L(C, n, N)$ is equal to the linear span of the coefficients (with respect to the variables $\mathbf{x}$ and $\mathbf{y})$ of the polynomials:

$$
\Delta_{N}^{\omega_{N}} \Delta_{N-1}^{\omega_{N-1}} \cdots \Delta_{2}^{\omega_{2}} \text { Source }(C)
$$

for all $\left(\omega_{2}, \ldots, \omega_{N-1}, \omega_{N}\right) \in \mathbb{N}^{N}$ such that $\omega_{2}+\cdots+\omega_{N-1}+\omega_{N} \leq d$.

Proof. For all $k \geq 0$ let $A_{k}$ be the linear span of the coefficients of $C_{\gamma}$ (with respect to the variables $\mathbf{x}$ and $\mathbf{y}$ ) for all $\gamma \in \mathbb{N}^{N}$ such that $\gamma_{1} \geq k$.

Then (8) simplifies into:

$$
\gamma_{j} C_{\gamma} \equiv \Delta_{j} C_{\gamma-\xi_{j}+\xi_{1}} \bmod A_{k+1} \mathbb{K}[\mathbf{x}, \mathbf{y}, \mathbf{z}] .
$$

Thanks to this formula, we can prove by induction on $d-k$, starting with $d-k=0$, that the following assertion is true for all $k$ between 0 and $d$ :

The vector space $A_{k}$ is equal to the linear span of the coefficients of the polynomials $(9)$ for all $\left(\omega_{2}, \ldots, \omega_{N-1}, \omega_{N}\right) \in \mathbb{N}^{N}$ such that $\omega_{2}+\cdots+$ $\omega_{N-1}+\omega_{N} \leq d-k$.

This assertion for $d-k=d$ is the assertion of the proposition.

\subsection{Removing redundancies: expression in the brackets}

Let $C$ be a homogeneous element of $\mathcal{A}$ of multidegree $(d, j, k)$. That is, $C$ belongs to

$$
\left(S^{d} S^{n} V \otimes\left(S^{j} V^{*} \otimes S^{j} V^{*}\right)^{S L_{2}} \otimes S^{k} V^{*}\right)^{S L(V)} .
$$

As representations of $S L(V)$ there is

$$
\left(S^{j} V^{*} \otimes S^{j} V^{*}\right)^{S L_{2}} \cong S^{(j, j)}\left(V^{*}\right)
$$

${ }^{3}$ After Hilbert [10] this term was coined by Sylvester. 
the Weyl module on $V^{*}$ indexed by the partition $(j, j)$ (see $[6]$ ). This is the set of forms in the variables $\mathbf{x}$ and $\mathbf{y}$ that can be expressed as a homogeneous polynomials of degree $j$ in the $N(N-1) / 2$ brackets $[i j]=x_{i} y_{j}-x_{j} y_{i}$ with $i<j$. When computing an element $C$ of $\mathcal{A}$ we should obtain such an expression, to avoid redundancy.

The brackets are not algebraically independent (except for $N \leq 3$ ). A linear basis for $S^{(d, d)}\left(V^{*}\right)$ are the products of brackets indexed by semi-standard tableaux. A product of brackets $\left[i_{1} j_{1}\right]\left[i_{2} j_{2}\right] \cdots\left[i_{d} j_{d}\right]$ (where for each $k$ we have $i_{k}<j_{k}$ ) is indexed by the unique $2 \times d$ array of integers

$$
\left[\begin{array}{llll}
j_{1} & j_{2} & \cdots & j_{d} \\
i_{1} & i_{2} & \cdots & i_{d}
\end{array}\right]
$$

whose columns are weakly increasing from left to right in lexicographic order. The array is a semi-standard tableau if each of its rows is weakly increasing from left to right.

An algorithm to express any $S L_{2}$-invariant polynomial as a linear combination of the products of brackets indexed by semi-standard tableaux is provided by $[12]$.

\subsection{Method}

We propose the following strategy for computing a small generating set for the linear span of the coefficients of $C$ where $C$ is Brill's covariant $B$ or Gaeta's covariant $G$ :

- Compute Source $(C)$ by applying the construction of Section 4 or Section 3 with $\mathbf{z}$ specialized at $(1,0,0, \ldots, 0)$.

- Express Source $(C)$ as a linear combination of the products of brackets indexed by standard tableaux. (There might exist a better way to compute the decomposition of Source $(C)$ in the brackets. For instance, for Brill's covariant, by means of the symbolic expression for the apolar covariant.).

- Apply the recurrent formulas presented in 5.1.

\subsection{A toy example: ternary quadratic forms}

It is well known, from the theory of quadratic forms, that a complex ternary quadratic form is totally decomposable if and only if the determinant of its matrix vanishes. We compute here Brill's covariant for the ternary quadratic forms to illustrate the methods presented in this section. We will obtain that Brill's equations are all proportional to the determinant of the matrix of the quadratic form.

The ternary quadratic form is:

$$
f(\mathbf{z})=a_{200} z_{1}^{2}+a_{020} z_{2}^{2}+a_{002} z_{3}^{2}+a_{110} z_{1} z_{2}+a_{101} z_{1} z_{3}+a_{011} z_{2} z_{3} .
$$

Its polars are $f^{(0)}(\mathbf{x}, \mathbf{z})=f(\mathbf{z}), f^{(2)}(\mathbf{x}, \mathbf{z})=f(\mathbf{x})$ and

$$
\begin{aligned}
f^{(1)}(\mathbf{x}, \mathbf{z})= & 2 a_{200} x_{1} z_{1}+2 a_{020} x_{2} z_{2}+2 a_{002} x_{3} z_{3} \\
& +a_{110}\left(x_{1} z_{2}+x_{2} z_{1}\right)+a_{101}\left(x_{1} z_{3}+x_{3} z_{1}\right)+a_{011}\left(x_{2} z_{3}+x_{3} z_{2}\right) .
\end{aligned}
$$


We evaluate them at $\mathbf{z}=(1,0,0)$ :

$$
\begin{aligned}
& f^{(0)}(\mathbf{x},(1,0,0))=a_{200}, \\
& f^{(1)}(\mathbf{x},(1,0,0))=2 a_{200} x_{1}+a_{110} x_{2}+a_{101} x_{3}, \\
& f^{(2)}(\mathbf{x},(1,0,0))=f(\mathbf{x}) .
\end{aligned}
$$

The evaluation at $\mathbf{z}=(1,0,0)$ of $f(t \mathbf{z}-f(\mathbf{z}) \mathbf{x}) / f(\mathbf{z})$ is therefore:

$$
t^{2}-\left(2 a_{200} x_{1}+a_{110} x_{2}+a_{101} x_{3}\right) t+a_{200} f(\mathbf{x})
$$

The symmetric power sum $p_{2}$ is obtained from the elementary symmetric polynomials as $e_{1}^{2}-2 e_{2}$. Set $g$ for $Q_{2}(f)(\mathbf{x} ;(1,0,0))$. We have:

$$
\begin{aligned}
g= & \left(2 a_{200} x_{1}+a_{110} x_{2}+a_{101} x_{3}\right)^{2}-2 a_{200} f(\mathbf{x}) \\
= & 2 a_{200}^{2} x_{1}^{2}+\left(a_{110}^{2}-2 a_{200} a_{020}\right) x_{2}^{2}+\left(a_{101}^{2}-2 a_{200} a_{002}\right) x_{3}^{2} \\
& +2 a_{200} a_{110} x_{1} x_{2}+2 a_{200} a_{101} x_{1} x_{3}+2\left(a_{110} a_{101}-a_{200} a_{011}\right) x_{2} x_{3}
\end{aligned}
$$

Therefore,

$$
\operatorname{Source}(B)=C_{\mathrm{Apo}, \mathbf{x}}(f, g)=2 f(\mathbf{y}) g(\mathbf{x})-f^{(1)}(\mathbf{x} ; \mathbf{y}) g^{(1)}(\mathbf{x} ; \mathbf{y})+2 f(\mathbf{x}) g(\mathbf{y})
$$

After decomposing in the brackets we obtain:

$$
\text { Source }(B)=-8[23]^{2} D
$$

where $[23]=x_{2} y_{3}-x_{3} y_{2}$ and $D$ is the determinant of the matrix of $f$ :

$$
D=a_{200} a_{020} a_{002}+\frac{1}{4} a_{110} a_{101} a_{011}-\frac{1}{4} a_{200} a_{011}^{2}-\frac{1}{4} a_{020} a_{101}^{2}-\frac{1}{4} a_{002} a_{110}^{2}
$$

After Proposition 8, Brill's equations span the same vector space as $D$ and the coefficients with respect to $\mathbf{x}$ and $\mathbf{y}$ of the following polynomials:

$$
\begin{array}{ll}
\Delta_{2} \text { Source }(B), & \Delta_{2}^{2} \operatorname{Source}(B), \quad \Delta_{3} \Delta_{2} \operatorname{Source}(B), \\
\Delta_{3} \text { Source }(B), & \Delta_{3}^{2} \operatorname{Source}(B) .
\end{array}
$$

We have:

and

$$
\Delta_{2}=a_{110} \frac{\partial}{d a_{200}}+2 a_{020} \frac{\partial}{d a_{110}}+a_{011} \frac{\partial}{d a_{101}}
$$

$$
\begin{aligned}
a_{110} \frac{\partial \operatorname{Source}(B)}{d a_{200}} & =-8[23]^{2}\left(a_{110} a_{020} a_{002}-\frac{1}{4} a_{110} a_{011}^{2}\right), \\
2 a_{020} \frac{\partial \operatorname{Source}(B)}{d a_{110}} & =-8[23]^{2}\left(\frac{1}{2} a_{020} a_{101} a_{011}-a_{002} a_{110} a_{020}\right), \\
a_{011} \frac{\partial \operatorname{Source}(B)}{d a_{101}} & =-8[23]^{2}\left(\frac{1}{4} a_{110} a_{011}^{2}-\frac{1}{2} a_{020} a_{101} a_{011}\right) .
\end{aligned}
$$

Therefore $\Delta_{2}$ Source $(B)=0$. Similarly we compute that $\Delta_{3} \operatorname{Source}(B)=0$. This shows that Brill's equations for the ternary quadratic form are all proportional to the determinant $D$. 


\subsection{Still more redundancies}

Let $C$ be either Gaeta's covariant $G$ or Brill's covariant $B$ and $(d, j, k)$ its multidegree. After expressing the homogeneous covariant $C$ as

$$
C=\sum c_{T, \gamma} T(\mathbf{x}, \mathbf{y}) \mathbf{z}^{\gamma}
$$

where the sum is carried over all monomials $T$ of degree $j$ in the brackets indexed by semi-standard tableaux and all monomials $\mathbf{z}^{\gamma}$ of degree $k$, we have obtained with the forms $c_{T, \gamma}$ a much smaller generating set for $L(C, n, N)$. We may expect these generators to be linearly independent. Explicit computations show the contrary: $\operatorname{dim} L(c, n, N)$ is still much smaller that the number of summands in (11). For instance, for $N=4$ with $n=3$, there are 4200 summands in (11) for $C=B$ but $\operatorname{dim} L(B, 3,4)=875$ (as reported in $[5,1])$.

For ternary forms, the explanation is known: Brill's covariant can be divided by the square of

$$
\left|\begin{array}{lll}
x_{1} & y_{1} & z_{1} \\
x_{2} & y_{2} & z_{2} \\
x_{3} & y_{3} & z_{3}
\end{array}\right|
$$

Indeed, proving this is the object of Gordan's paper [9]. One can show that the same holds for Gaeta's covariant. Therefore, for ternary forms, there exist polynomial covariants $\widehat{C}$ (for $C=B$ or $G$ ) such that:

$$
C=\left|\begin{array}{lll}
x_{1} & y_{1} & z_{1} \\
x_{2} & y_{2} & z_{2} \\
x_{3} & y_{3} & z_{3}
\end{array}\right|^{2} \cdot \widehat{C}
$$

A symbolic form for $\widehat{B}$ is provided by [4] for $n=N=3$ and by Gordan [9] for $N=3$, all $n$.

The smaller covariant $\widehat{C}$ can be decomposed into:

$$
\widehat{C}=\sum_{T, \gamma} \widehat{c}_{T, \gamma} T(\mathbf{x}, \mathbf{y}) \mathbf{z}^{\gamma}
$$

where the sum is carried over all $T$ monomials of degree $j-2$ in the brackets and all $\mathbf{z}^{\gamma}$ monomials of degree $k-2$. The forms $\widehat{c}_{T, \gamma}$ give a still smaller generating system for $L(C, n, 3)$. Explicit computation show that the cardinal of this generating set coincides with $\operatorname{dim} L(B, n, 3)$ for $C=B$ and $n=4$ and $n=5$. (For $n=3$ the generating set has 45 elements but $\operatorname{dim} L(B, 3,3)=35$.) After this we can expect that for all $n>3$,

$$
\operatorname{dim} L(B, n, 3)=\left(\begin{array}{l}
n \\
2
\end{array}\right)^{2}\left(n^{2}-n-1\right) .
$$

Note that the method presented in Section 5.3 is easily adapted to this simplification. It is enough to observe that for $N=3$,

$$
\operatorname{Source}(\widehat{C})=\operatorname{Source}(C) /[23]^{2} \text {. }
$$


It would be interesting to study how the above factorization property of $B$ and $G$ exhibited for ternary forms generalizes to forms of higher arity, and how to use it for more efficient computations. Brill's covariant and Gaeta's covariant belong to the ideal $I$ generated by the maximal minors of the matrix

$$
\left[\begin{array}{llll}
x_{1} & x_{2} & \cdots & x_{N} \\
y_{1} & y_{2} & \cdots & y_{N} \\
z_{1} & z_{2} & \cdots & z_{N}
\end{array}\right]
$$

since, by construction, they vanish for all specializations of $\mathbf{x}, \mathbf{y}$ and $\mathbf{z}$ at three coplanar vectors. We conjecture that Brill's covariant and Gaeta's covariant actually belong to the square of the ideal $I$.

\section{Acknowledgment}

I would like to thank Maria Emilia Alonso and Mercedes Rosas for their careful reading of this paper.

\section{References}

[1] Emmanuel Briand. Polynômes multisymétriques, 2002. Doctoral thesis. Université de Rennes 1.

[2] Emmanuel Briand. Brill's equations for the subvariety of factorizable forms. In Laureano González-Vega and Tomás Recio, editors, Actas de los Encuentros de Álgebra Computacional y Aplicaciones: EACA 2004, pages 59-63, 2004. Santander. Available at http://emmanuel.jean.briand.free.fr/publications/

[3] Alexander von Brill. Über symmetrische Functionen von Variabelnpaaren. Nachrichten von der Königlichen Gesellschaft der Wissenschaften und der Georg-AugustUniversität zu Göttingen, 20:757-762, 1893.

[4] Jaydeep V. Chipalkatti. Decomposable ternary cubics. Experiment. Math., 11(1):6980, 2002.

[5] John Dalbec. Multisymmetric functions. Beiträge zur Algebra und Geometrie, 40(1):27-51, 1999.

[6] William Fulton. Young tableaux. London Mathematical Society Student Texts 35. Cambrige University Press, 1997.

[7] Federico Gaeta, 1999. Personal communication.

[8] I.M. Gel'fand, M.M. Kapranov, and A.V. Zelevinsky. Discriminants, resultants, and multidimensional determinants. Birkhäuser Boston Inc., Boston, MA, 1994.

[9] Paul Gordan. Das Zerfallen der Curven in gerade Linien. Math. Ann., 45:410-427, 1894.

[10] David Hilbert. Theory of algebraic invariants. Cambridge University Press, Cambridge, 1993. Translated from the German and with a preface by Reinhard C. Laubenbacher, Edited and with an introduction by Bernd Sturmfels. 
[11] Michael F. Singer and Felix Ulmer. Linear differential equations and products of linear forms. J. Pure Appl. Algebra, 117/118:549-563, 1997. Algorithms for algebra (Eindhoven, 1996).

[12] Bernd Sturmfels. Algorithms in invariant theory. Texts and Monographs in Symbolic Computation. Springer-Verlag, Wien, New York, 1993.

Emmanuel Briand

Universidad de Sevilla

Departamento de Álgebra

Facultad de Matemáticas

Aptdo. de Correos 1160

E-41080, Sevilla, Spain 